Method We designed the prompt guide in response to the findings from the Adverse Drug Reactions in Children: Qualitative study (ADRIC: QUAL) that identified parents unmet information and communication needs following a suspected ADR in their child. The prompt guide was developed by the ADRIC: QUAL and wider ADRIC study team; reviewed by parent expert advisory groups and expert advisory groups at the Medicines and Health care products Regulatory Agency (MHRA) and the Royal College of Paediatrics and Child Health (RCPCH) medicines committee before being finalised. The prompt guide was piloted at a regional children's hospital and it is the findings of the pilot study that are reported here.

We identified suspected ADRs in children through daily ward visits. Treating clinicians used the prompt guide during routine conversations with parents about that suspected ADR. Clinicians then took part in a short structured interview.

Results The prompt guide was used 17 times by nurses $(\mathrm{n}=9)$, specialist nurses $(n=4)$, and doctors $(n=4)$.

Clinicians who used the prompt guide felt it was easy to use; was helpful in structuring and pacing the discussion; ensured all relevant topics were covered,; and empowered parents.

An unexpected finding was confusion about whether a child had experienced a suspected ADR and this influenced whether discussions with families took place. Where discussions did take place, there was confusion about who communicated with parents. While doctors felt nurses were often the first to recognise, and therefore communicate with parents about suspected ADRs, few nurses recognised their role in this process and their accounts suggest that they are poorly supported in this role.

Conclusion Optimal communication with parents about suspected ADRs in children depends on clinicians recognising a suspected ADR. Nurses potentially play an important role in communicating with families in such situations but they do not always recognise their role and there maybe unmet training needs.

\section{G26 PARENTS' EXPERIENCES OF ADMINISTERING AND MANIPULATING MEDICINES FOR CHILDREN WITH LONG TERM CHRONIC CONDITIONS}

\begin{abstract}
${ }^{1,2} \mathrm{~J}$ Arnott, ${ }^{2} \mathrm{R}$ Richey, ${ }^{1,2}{ }^{2} \mathrm{M}$ Peak, ${ }^{2} \mathrm{AJ}$ Nunn, ${ }^{3,4} \mathrm{MA}$ Turner. ${ }^{1}$ School of Health, University of Central Lancashire, Preston, UK; ${ }^{2}$ Research and Development, Alder Hey Children's NHS Foundation Trust, Liverpool, UK; ${ }^{3}$ Institute of Translational Health, University of Liverpool, Liverpool, UK; ${ }^{4}$ Department of Women's and Children's Health, Liverpool Women's NHS Foundation Trust, Liverpool, UK
\end{abstract}

\subsection{6/archdischild-2015-308599.26}

\section{Aims}

1. To explore the experiences and methods used by parents to administer long term medicines to their children.

Methods Semi structured qualitative interviews with parents of children who are prescribed long term medication for a chronic condition. Interviews were audio recorded and transcribed. Analysis was inductive and informed by the principles of grounded theory and constant comparison method.

Results Semi structured interviews with the parents of 13 $(\mathrm{N}=12)$ children between 5 to 11 years; female $(\mathrm{n}=5)$ and male $(\mathrm{n}=8)$. The sample is currently being extending to include the parents of up to 20 children.

Parent's accounts were variable. Some parents reported no problems administering medicines to their child but other parents described prolonged 'battles'. Taste was cited as the main reason for a child refusing a medicine. Some methods used by parents to encourage children to comply with medication, such as rewards and reasoning are commonly used by parents giving short term medicines but these methods needed to be regularly adapted over time as their effect wore off. Parents also described ordering medicines, giving two medicines together or missing medicine doses in order to reduce 'battles' and increase compliance.

Parents were confident in manipulating medicines (cutting, crushing or splitting tablets etc.), but had concerns about controlling the dose their child received e.g. tablets crumbling. Similarly, parents did not favour disguising medicines in food or drink as they felt this method did not work, risked losing the trust of their child, and their child potentially refusing that particular food or drink in the future. Furthermore, parents had concerns about controlling the dose of a medicine using this method.

Conclusion Some parents described 'battles' over administering medicine to their children that had a significant and negative impact on daily family life. Taste was the major barrier to compliance. Methods perceived as favourable by clinicians, such as disguising medicines in food and drink, were not favoured by parents who employed a range of alternative strategies, some of which involved altering medicine regimens and was not supported by evidence.

\section{G27 SOCIAL INFLUENCES ON PARENTS' HEALTH SERVICE USE WHEN THEIR CHILD IS SICK: BARRIERS TO TIMELY TREATMENT?}

SJ Neill. School of Health, University of Northampton, Northampton, UK

\subsection{6/archdischild-2015-308599.27}

Acute childhood illness is surrounded by uncertainty and anxiety about the nature of the illness and when to seek professional help (Neill 2010). Social influences on health service use are complex but need to be understood if services are to address barriers to timely treatment.

Aim To explore the impact of social influences on parents' health service use during acute childhood illness.

Methods This paper draws findings from 3 projects which explored parents' decision making during acute childhood illness at home. These studies include: a grounded theory study with 15 families (Neill 2008), a social marketing project involving 23 parents in focus groups (Spencer and Neill 2013) and a modified grounded theory study with 27 parents using focus groups and interviews (Jones et al. 2013, Neill et al. 2014).

Results Three themes emerged in the data from all 3 projects: Social expectations; Family and social support; and Service design and its impact on access to services. Social expectations shape how parents perceive they are expected to act as mly 'good parents', including an expectation that they will use services appropriately, creating anxiety about attending too soon or too late. Family and social support provides emotional and practical support, not usually advice on how to manage the illness, contrary to professionals' assumptions about the role of extended families. Services are not designed with the child and family in mind: systems create delay, increasing parents' anxiety; difficulties in accessing services; limited continuity; and confusion about which service to use.

Conclusion A wide range of social factors influence parents' help seeking in childhood illness. When parents decide to 